\title{
Efeito do diluente pigpel na qualidade do sêmen suíno refrigerado em diferentes temperaturas
}

\section{Effect of pigpel extender on the boar semen quality stored at different temperatures}

\author{
Flavio Juliano ${ }^{1}$; Carolina Gonçalves Serret ${ }^{1}$; Augusto Schneider ${ }^{2}$; Viviane Rohrig \\ Rabassa ${ }^{3}$; Carlos Eduardo Wayne Nogueira"; Telmo Vidor ${ }^{5}$; Thomaz Lucia Junior ${ }^{4}$; \\ João Carlos Deschamps ${ }^{6}$; Ivan Bianchi'; Marcio Nunes Corrêa ${ }^{4 *}$
}

\section{Resumo}

Este estudo avaliou a qualidade do sêmen suíno preservado em diferentes temperaturas e meios de conservação no diluente PIGPEL5, utilizando gema de ovo como protetor externo de membrana, e no diluente PIGPEL5Plus, utilizando lipoproteína de baixa densidade (LDL) como protetor. No experimento 1 , as doses de sêmen foram conservadas em refrigerador de sêmen a 5 e $17^{\circ} \mathrm{C}$ nos diluentes PIGPEL5 e PIGPEL5Plus e comparadas ao diluente BTS a $17^{\circ} \mathrm{C}$. Já no experimento 2, foi comparada a conservação do sêmen nos diluentes PIGPEL5 e PIGPEL5Plus em geladeira convencional ou em refrigerador de sêmen a $5{ }^{\circ} \mathrm{C}$. Observou-se que o uso de diluidores com crioprotetor extracelular (PIGPEL5 e PIGPEL5Plus) apresentou maiores $(\mathrm{p}<0,05)$ resultados de motilidade com relação ao BTS até $48 \mathrm{~h}$. Além do mais, o uso de crioprotetor extracelular foi benéfico para a manutenção da integridade e morfologia normais a temperatura de $5{ }^{\circ} \mathrm{C}$ em níveis similares aos diluidores a $17^{\circ} \mathrm{C}$. Não houve diferença $(\mathrm{p}>0,05)$ na motilidade, integridade de membrana e morfologia para a substituição da gema de ovo (PIGPEL5) pela LDL (PIGPEL5Plus). No experimento 2, também não houve diferença na motilidade, integridade de membrana e morfologia espermática entre os diluentes e formas de acondicionamento testados até $72 \mathrm{~h}(\mathrm{p}>0,05)$. O acondicionamento do sêmen no diluente PIGPEL5 em geladeira doméstica $\left(5^{\circ} \mathrm{C}\right)$ proporcionou padrões de qualidade espermática de acordo com o recomendado para a IA.

Palavras-chave: Sêmen, gema de ovo, lipoproteína de baixa densidade, temperatura

\begin{abstract}
This study aim to evaluate the quality of boar semen stored at different temperatures and media on PIGPEL5 extender, using egg yolk as an external membrane protector, and on PIGPEL5Plus, containing low density lipoprotein (LDL) as protector. In the experiment 1 , the semen doses were stored in a semen container at 5 and $17{ }^{\circ} \mathrm{C}$ and the results were compared with semen stored at $17{ }^{\circ} \mathrm{C}$ in BTS extender. In the experiment 2 , the doses with the PIGPEL5 and PIGPEL5Plus extenders were stored in a commercial
\end{abstract}

\footnotetext{
Médico Veterinário, Mestre em Veterinária

2 Médico Veterinário, Doutorando em Biotecnologia Agrícola, UFPel

3 Médica Veterinária, Doutoranda em Veterinária, UFPel

${ }^{4}$ Médico Veterinário, Dr., Professor Adjunto, Faculdade de Veterinária, UFPel. Núcleo de Pesquisa, Ensino e Extensão em Pecuária, Universidade Federal de Pelotas - UFPel; Departamento de Clínicas Veterinária, Campus Universitário, s/nº; CEP: 96010-900, Pelotas/RS. Site: http://www.ufpel.edu.br/nupeec. E-mail: nupeec@ufpel.edu.br

5 Médico Veterinário, Dr., Professor Associado, Faculdade Veterinária, UFPel

6 Médico Veterinário, Dr., Professor Titular, Faculdade de Veterinária, UFPel

* Autor para correspondência
} 
refrigerator and in semen container, both at $5{ }^{\circ} \mathrm{C}$. The use of extenders with extracellular cryoprotectant (PIGPEL5 e PIGPEL5Plus) showed higher $(\mathrm{p}<0.05)$ motility values in comparison to BTS until $48 \mathrm{~h}$. Furthermore, the use of extracellular cryoprotectant was good to maintenance of membrane integrity and normal morphology at $5{ }^{\circ} \mathrm{C}$ similarly to $17{ }^{\circ} \mathrm{C}$ extenders. There was no difference $(\mathrm{p}>0.05)$ in the motility, membrane integrity and morphology for the substitution of egg yolk (PIGPEL5) for LDL (PIGPEL5Plus). In experiment 2, also there was no difference $(\mathrm{p}>0.05)$ in motility, membrane integrity and morphology between extenders and type of container until $72 \mathrm{~h}$. The conservation of semen on PIGPEL5 extender and commercial refrigerator $\left(5^{\circ} \mathrm{C}\right)$ allowed patterns of sperm quality according recommended for AI.

Key words: Semen, egg yolk, low density lipoprotein, temperature

\section{Introdução}

A grande maioria das inseminações artificiais (IA) realizadas em suínos utiliza sêmen diluído e acondicionado a temperaturas entre 15 e $18{ }^{\circ} \mathrm{C}$ por um período de máximo de 5 dias, sendo $85 \%$ destas realizadas no dia da coleta do sêmen ou no dia seguinte (JOHNSON et al., 2000). O diluente de sêmen suíno mais utilizado é o Beltsville Thawing Solution (BTS) (PURSEL; JHONSON, 1975), inicialmente utilizado para congelamento de sêmen e, posteriormente, adaptado para o acondicionamento em refrigerador a temperaturas entre 15 e $18^{\circ} \mathrm{C}$ por até 3 dias. No entanto, os refrigeradores utilizados apresentam um custo elevado, principalmente para pequenas unidades de produção e quando submetidos às variações da temperatura ambiente apresentam dificuldade em estabilizar a temperatura interna na faixa desejada. Além disso, a estocagem do sêmen limita o transporte, restringindo o tempo de vida útil da célula espermática, visto que não impede a atividade metabólica celular, nem o crescimento bacteriano (ALTHOUSE; LU, 2005).

Alguns diluentes foram desenvolvidos a fim de possibilitar o aumento no tempo de estocagem para 5 a 7 dias (LEVIS, 2000), ou mesmo possibilitar o armazenamento do sêmen a $5{ }^{\circ} \mathrm{C}$ a fim de conserválo em geladeira doméstica (CORRÊA et al., 2004, 2006). No entanto, o espermatozóide suíno é muito susceptível ao choque térmico, o que ocorre quando o ejaculado fresco é rapidamente resfriado a temperaturas abaixo de $15{ }^{\circ} \mathrm{C}$, resultando em perda da viabilidade de um número elevado de espermatozóides (JOHNSON et al., 2000), alterações em componentes essenciais das células espermáticas e perda da qualidade do ejaculado quando comparado ao sêmen fresco (AMIRAT et al., 2004; WATSON, 2000).

A gema de ovo é um dos crioprotetores externos mais utilizados em protocolos para congelação de sêmen ou quando se utiliza sêmen resfriado abaixo de $15^{\circ} \mathrm{C}$ (CORRÊA et al, 2004). Entretanto, contrapondo seu efeito benéfico, a gema de ovo é composta por substâncias que dificultam a respiração celular, interferindo no sistema de transporte de elétrons nas mitocôndrias, podendo diminuir a motilidade espermática, além de ser um risco de contaminação por microorganismos (BOUSSEAU et al., 1998). O efeito crioprotetor da gema de ovo é dado pela presença da lipoproteína de baixa densidade (LDL), a qual associa-se à membrana plasmática, protegendo o espermatozóide do choque térmico (BERGERON et al., 2004; DEMANIOWICZ; STRZEZEK, 1996; WATSON, 1981). A LDL forma uma película interfacial entre os ácidos graxos da membrana e a água (BERGERON et al., 2004), promovendo a entrada e prevenindo a saída de fosfolipídios e colesterol na membrana e formando um complexo com as proteínas do plasma seminal de modo que estas não fiquem disponíveis para atuar na membrana (MOUSSA et al., 2002). A prevenção do efluxo de fosfolipídios e colesterol confere à célula espermática maior resistência ao choque térmico (GRAHAM; FOOTE, 1987; TRIMECHE et al., 1996). Neste contexto, a LDL pode aumentar a resistência contra o choque térmico, permitindo uma maior qualidade espermática de ejaculados acondicionados a baixas temperaturas. 
O objetivo deste estudo foi avaliar o desempenho dos diluentes PIGPEL5 (com gema de ovo) e PIGPEL5Plus (com LDL) em preservar a qualidade do sêmen suíno resfriado na forma líquida em diferentes temperaturas $\left(5\right.$ e $\left.17^{\circ} \mathrm{C}\right)$, e sob diferentes formas de estocagem.

\section{Material e métodos}

Este trabalho foi dividido em dois experimentos, sendo utilizados ejaculados provenientes de três machos suínos F1 (Landrace x Large White) com fertilidade conhecida e aproximadamente 12 meses de idade.

\section{Experimento 1}

No experimento 1 foram realizadas quatro coletas de cada macho. Após a coleta o ejaculado foi diluído em condições isotérmicas em 3 diluentes: BTS (PURSEL; JHONSON, 1975), PIGPEL5 (CORRÊA et al., 2004) e PIGPEL5Plus. O diluente PIGPEL5 utilizou gema de ovo como componente protetor extracelular na concentração de $2 \%$, já o diluente PIGPEL5Plus teve a gema de ovo substituída pela LDL em quantidade equivalente a gema de ovo. A LDL foi purificada através do protocolo descrito por Moussa et al. (2002). As doses (100 mL cada), com uma concentração de $3,5 \times 10^{9}$ espermatozóides, foram submetidas a um tempo inicial de resfriamento de $3 \mathrm{~h}$ a uma temperatura variando entre $22-24{ }^{\circ} \mathrm{C}$ (CORRÊA et al., 2001) e então, acondicionadas. As doses de sêmen que foram diluídas em BTS foram acondicionadas em refrigerador à temperatura de $17{ }^{\circ} \mathrm{C}$, enquanto que as doses diluídas no PIGPEL5 e PIGPEL5Plus foram acondicionadas em refrigeradores à temperatura de 5 e $17^{\circ} \mathrm{C}$, ou seja, ambos foram submetidas a duas temperaturas.

\section{Experimento 2}

No experimento 2 foram utilizados quatro ejaculados de cada macho, que após avaliação foram diluídos nos diluentes PIGPEL5 e PIGPEL5Plus e acondicionados à temperatura de $5{ }^{\circ} \mathrm{C}$ em refrigerador específico para sêmen ou em geladeira doméstica convencional.

Os refrigeradores de sêmen que serviram para o acondicionamento das doses durante o estudo eram dotados de termostatos reguláveis, conferindo, dessa forma, a temperatura desejada de estocagem, conforme o diluente utilizado. A geladeira doméstica utilizada durante o experimento 2 não sofreu modificações quanto a sua capacidade de preservação da temperatura interna, sendo regulada simplesmente para manter a temperatura em torno de $5{ }^{\circ} \mathrm{C}$, mantendo assim as características de uma geladeira doméstica convencional. A temperatura do ambiente interior da geladeira doméstica foi aferida diariamente às $8 \mathrm{~h}, 12 \mathrm{~h}$ e $16 \mathrm{~h}$, com termômetro digital de precisão (Incoterm $\AA$ ) variando entre 3,4 e $5,9^{\circ} \mathrm{C}$.

\section{Análise e conservação do sêmen}

Para diluição, somente foram utilizados ejaculados que apresentassem no mínimo $70 \%$ de espermatozóides móveis logo após a coleta. Todos os tratamentos estavam representados em cada ejaculado coletado de cada macho doador, ou seja, o ejaculado de cada animal foi dividido em alíquotas, para cada um dos tratamentos a serem testados nos respectivos experimentos.

O sêmen foi avaliado quanto à motilidade, integridade de membrana emorfologia. As avaliações espermáticas foram realizadas imediatamente após a diluição (hora zero "0 h") e ao longo do período de conservação (24, 48 e 72 h).

A avaliação da motilidade (0 a 100\%) foi realizada sempre pelo mesmo técnico em um microscópio óptico em aumento de 200x (TARDIF et al., 1999). Para avaliação era utilizada uma amostra de $2 \mathrm{~mL}$ do sêmen e incubado em tubos cônicos de $15 \mathrm{~mL}$ em banho maria a $37{ }^{\circ} \mathrm{C}$ durante $10 \mathrm{~min}$. Após a incubação uma amostra de $10 \mu$ do sêmen era 
depositada entre lâmina e lamínula previamente aquecidas à $37^{\circ} \mathrm{C}$ para avaliação no microscópio.

A integridade da membrana espermática foi avaliada através do teste do choque hiposmótico (CHIPO) (VAZQUEZ et al., 1997). Para tal, duas soluções de diferente osmolaridades foram usadas, uma com $75 \mathrm{mOsm} / \mathrm{L}$ (hiposmótica) e uma com $312 \mathrm{mOsm} / \mathrm{L}$ (isosmótica). Para a realização do teste utilizou-se $900 \mu \mathrm{l}$ de solução hiposmótica e $100 \mu \mathrm{l}$ de sêmen em eppendorf de $1,5 \mathrm{~mL}$, o qual foi homogeinizado e incubado em banho-maria à $37{ }^{\circ} \mathrm{C}$ durante $5 \mathrm{~min}$. $\mathrm{O}$ mesmo procedimento foi feito com a solução isosmótica. No teste do CHIPO, espermatozóides com cauda enrolada na solução hiposmótica indicam que a célula estava íntegra, pois, devido a baixa osmolaridade da solução a água penetra na célula a fim de equilibrar as concentrações ocasionando a reação característica que é o fenômeno da cauda enrolada. É realizada também a avaliação na solução isosmótica, a fim de quantificar o percentual de células com cauda enrolada devido a defeitos patológicos. Dessa forma, após a incubação, através de microscopia óptica com contraste de fases, em aumento de 400 vezes, procedeu-se à contagem de 100 espermatozóides incubados em cada tipo de solução (isosmótica e hiposmótica), registrando o número de células que apresentaram cauda enrolada. $\mathrm{O}$ valor que foi utilizado para análise do CHIPO foi à diferença entre o número de espermatozóides com caudas enroladas observadas na solução hiposmótica e isosmótica.

A avaliação da morfologia espermática consistiu em verificar todas as alterações de acrossoma, cabeça, peça intermediária e cauda dos espermatozóides. O procedimento de preparação do teste iniciou com a deposição de 3 gotas de sêmen em $2 \mathrm{~mL}$ de formol salina (fixador) em iguais temperaturas. Após suave homogeinização, uma gota da amostra foi depositada sobre uma lâmina de microscopia, adicionada uma gota de corante eosina e coberta por uma lamínula. A análise morfológica dos espermatozóides foi feita em microscópio com contraste de fases em aumento de 1000x, com utilização de uma gota de óleo de imersão sobre a lâmina, onde se contou um total de 100 células por lâmina (WOELDERS, 1991; COLÉGIO BRASILEIRO DE REPRODUÇÃO ANIMAL, 1998). Para avaliação estatística foi utilizado somente o percentual de células classificadas como de morfologia normal.

\section{Análise estatística}

Os resutados são descritos como média \pm desvio padrão da média. Em cada experimento, para as variáveis dependentes: motilidade, integridade de membrana e morfologia foi gerada uma análise de variância pelo modelo linear através de medidas repetidas, a fim de isolar o efeito de cada coleta e de cada macho sobre os parâmetros avaliados. Para isso foram consideradas as variáveis independentes e possíveis interações entre elas. A comparação entre as médias foi feita através do teste LSD (Least Significance Difference). A análise estatística foi feita utilizando o programa Statistix ${ }^{\circledR}$ (2003).

\section{Resultados e discussão}

\section{Experimento 1}

A motilidade do sêmen no diluente PIGPEL5 a $5{ }^{\circ} \mathrm{C}$ foi maior que o diluente BTS às $48(65,0$ $\pm 6,7 \%$ e $45,0 \pm 24,3 \%)$ e $72 \mathrm{~h}(65,0 \pm 5,2 \%$ e $33,3 \pm 26,0 \%$ ), mantendo valores aceitáveis de motilidade para sêmen refrigerado de acordo com as recomendações para a espécie (CBRA, 1998). Além disso, o diluente PIGPEL5 à $5{ }^{\circ} \mathrm{C}$ foi o que apresentou menor variabilidade durante as $72 \mathrm{~h}$ de conservação, como observado pelo menor desvio padrão da média (Tabela 1). Assim, observou-se que em geral o uso de diluidores com crioprotetor extracelular (PIGPEL5 e PIGPEL5Plus) apresentou melhores resultados de motilidade com relação ao BTS até 48 h (Tabela 1), conforme já demonstrado por Corrêa et al. (2006). 
Tabela 1. Percentual de espermatozóides móveis durante o período de conservação (média \pm desvio padrão)

\begin{tabular}{cccccc}
\hline \multirow{2}{*}{ Diluentes } & \multirow{2}{*}{${\text { Temperatura }\left({ }^{\circ} \mathbf{C}\right)}^{*}$} & \multicolumn{4}{c}{ Células móveis (\%) nos períodos de conservação } \\
\cline { 3 - 6 } & & $\mathbf{0 ~ h}$ & $\mathbf{2 4} \mathbf{~ h}$ & $\mathbf{4 8 ~ h}$ & $\mathbf{7 2 ~ h}$ \\
\hline BTS & 17 & $70,8 \pm 5,2^{\mathrm{a}}$ & $55,0 \pm 21,9^{\mathrm{b}}$ & $45,0 \pm 24,3^{\mathrm{b}}$ & $33,3 \pm 26,0^{\mathrm{b}}$ \\
PIGPEL5 & 17 & $75,8 \pm 5,2^{\mathrm{a}}$ & $74,2 \pm 5,2^{\mathrm{a}}$ & $53,3 \pm 32,3^{\mathrm{ab}}$ & $26,7 \pm 28,2^{\mathrm{b}}$ \\
PIGPEL5Plus & 17 & $75,0 \pm 6,7^{\mathrm{a}}$ & $74,2 \pm 6,7^{\mathrm{a}}$ & $68,3 \pm 9,4^{\mathrm{a}}$ & $50,8 \pm 27,5^{\mathrm{ab}}$ \\
PIGPEL5 & 5 & $75,8 \pm 5,2^{\mathrm{a}}$ & $69,2 \pm 5,2^{\mathrm{ab}}$ & $65,0 \pm 6,7^{\mathrm{a}}$ & $65,0 \pm 5,2^{\mathrm{a}}$ \\
PIGPEL5Plus & 5 & $75,8 \pm 5,2^{\mathrm{a}}$ & $59,9 \pm 17,3^{\mathrm{ab}}$ & $58,2 \pm 10,8^{\mathrm{ab}}$ & $54,2 \pm 13,1^{\mathrm{ab}}$ \\
\hline
\end{tabular}

a, b Médias com letras diferentes na mesma coluna diferem estatisticamente $(p<0,05)$

A integridade de membrana e morfologia espermática não diferiu $(\mathrm{p}>0,05)$ com relação ao uso de crioprotetores extracelulares (Tabela 2 e 3 ). Os valores encontrados para a integridade de membrana e morofologia no presente estudo são compatíveis com resultados anteriores (VAZQUEZ et al., 1997; CORRÊA et al., 2006). Por outro lado, apesar de danos na morfologia espermática serem descritos quando o sêmen suíno é acondicionado abaixo de $15^{\circ} \mathrm{C}$ (PURSEL; JOHNSON; SCHULMAN, 1972; PURSEL; JOHNSON; RAMPACEK, 1972, 1973), no presente estudo os valores para morfologia estão de acordo com os padrões recomendados para a espécie (CBRA, 1998). Portanto, pode-se concluir que o uso de crioprotetor extracelular foi benéfico para a manutenção da integridade e morfologia normais a temperatura de $5{ }^{\circ} \mathrm{C}$ em níveis similares aos diluidores a $17^{\circ} \mathrm{C}$.

Tabela 2. Integridade funcional da membrana espermática (CHIPO) nos diferentes tratamentos e períodos de conservação de sêmen (média \pm desvio padrão)

\begin{tabular}{llllll}
\hline \multirow{2}{*}{ Diluentes } & \multirow{2}{*}{ Temperatura $\left({ }^{\circ} \mathrm{C}\right)$} & \multicolumn{5}{l}{ CHIPO nos períodos de conservação } \\
\cline { 3 - 6 } & & $0 \mathrm{~h}$ & $24 \mathrm{~h}$ & $48 \mathrm{~h}$ & $72 \mathrm{~h}$ \\
\hline BTS & 17 & $48,3 \pm 8,2^{\mathrm{a}}$ & $46,2 \pm 3,9^{\mathrm{a}}$ & $43,7 \pm 4,1^{\mathrm{ab}}$ & $33,0 \pm 4,1^{\mathrm{b}}$ \\
PIGPEL5 & 17 & $48,1 \pm 10,7^{\mathrm{a}}$ & $48,3 \pm 7,5^{\mathrm{a}}$ & $44,3 \pm 4,8^{\mathrm{ab}}$ & $31,8 \pm 1,4^{\mathrm{b}}$ \\
PIGPEL5Plus & 17 & $49,8 \pm 13,1^{\mathrm{a}}$ & $45,2 \pm 7,1^{\mathrm{a}}$ & $52,5 \pm 13,2^{\mathrm{a}}$ & $40,0 \pm 11,6^{\mathrm{a}}$ \\
PIGPEL5) & 5 & $46,0 \pm 11,7^{\mathrm{a}}$ & $43,0 \pm 2,6^{\mathrm{a}}$ & $42,5 \pm 7,2^{\mathrm{b}}$ & $34,2 \pm 3,6^{\mathrm{ab}}$ \\
PIGPEL5Plus & 5 & $46,3 \pm 11,9^{\mathrm{a}}$ & $43,0 \pm 3,9^{\mathrm{a}}$ & $42,7 \pm 2,9^{\mathrm{b}}$ & $35,5 \pm 4,7^{\text {ab }}$ \\
\hline
\end{tabular}

${ }^{\mathrm{a}, \mathrm{b}}$ Médias com letras diferentes na mesma coluna diferem estatisticamente $(\mathrm{p}<0,05)$

Tabela 3. Espermatozóides com morfologia normal (\%) nos diferentes tratamentos e períodos de conservação do sêmen (média \pm desvio padrão)

\begin{tabular}{llllll}
\hline \multirow{2}{*}{ Diluentes } & \multirow{2}{*}{ Temperatura $\left({ }^{\circ} \mathrm{C}\right)$} & \multicolumn{5}{l}{ Morfologia espermática normal (\%) } \\
\cline { 3 - 6 } & & $0 \mathrm{~h}$ & $24 \mathrm{~h}$ & $48 \mathrm{~h}$ & $72 \mathrm{~h}$ \\
\hline BTS & 17 & $87,3 \pm 13,5^{\mathrm{abc}}$ & $84,8 \pm 17,4^{\mathrm{a}}$ & $79,3 \pm 21,8^{\mathrm{a}}$ & $82,2 \pm 18,5^{\mathrm{a}}$ \\
PIGPEL5 & 17 & $84,2 \pm 16,7^{\mathrm{c}}$ & $86,2 \pm 17,1^{\mathrm{a}}$ & $88,8 \pm 13,1^{\mathrm{a}}$ & $87,6 \pm 12,6^{\mathrm{a}}$ \\
PIGPEL5Plus & 17 & $88,6 \pm 11,8^{\mathrm{a}}$ & $91,1 \pm 8,2^{\mathrm{a}}$ & $81,1 \pm 19,6^{\mathrm{a}}$ & $91,2 \pm 9,9^{\mathrm{a}}$ \\
PIGPEL5) & 5 & $85,2 \pm 16,4^{\mathrm{bc}}$ & $85,3 \pm 18,7^{\mathrm{a}}$ & $83,1 \pm 14,3^{\mathrm{a}}$ & $84,7 \pm 22,2^{\mathrm{a}}$ \\
PIGPEL5Plus & 5 & $87,7 \pm 13,3^{\mathrm{ab}}$ & $87,8 \pm 12,3^{\mathrm{a}}$ & $81,3 \pm 20,1^{\mathrm{a}}$ & $88,4 \pm 13,4^{\mathrm{a}}$ \\
\hline
\end{tabular}

${ }^{a, b, c}$ Médias com letras diferentes na mesma coluna diferem estatisticamente $(p<0,05)$ 
A ausência de diferença na motilidade, integridade de membrana e morfologia para a substituição da gema de ovo (PIGPEL5) pela LDL (PIGPEL5Plus) pode ser devido à baixa inclusão da LDL. Isto sugere que um percentual maior de inclusão deva ser testado para temperaturas menores de conservação, como já foi demonstrado por Moussa et al. (2002), Hu et al. (2006) e Varela Junior et al. (2008) com uso de sêmen congelado, os quais observaram um incremento na motilidade e integridade de membrana com a substituição de gema de ovo por quantidades maiores de LDL.

Uma das vantagens do armazenamento de sêmen a $5{ }^{\circ} \mathrm{C}$ é a redução da produção de catabólitos em função do menor metabolismo celular, havendo, portanto, menor risco de proliferação bacteriana quando comparada a conservação a $15{ }^{\circ} \mathrm{C}$ (ALTHOUSE; LU, 2005). Assim, a ausência de diferença na qualidade seminal entre os diluentes testados a 5 e $17{ }^{\circ} \mathrm{C}$ também é benéfica, pois a conservação em baixa temperatura permite a redução da proliferação de patógenos em longos períodos de conservação.

\section{Experimento 2}

A temperatura interna na geladeira doméstica (média \pm desvio padrão) nos três momentos de avaliação foi: $4,2^{\circ} \mathrm{C} \pm 0,8$ as $8 \mathrm{~h}, 4,5^{\circ} \mathrm{C} \pm 1,2$ as 12 h e $4,6^{\circ} \mathrm{C} \pm 1,3$ as $16 \mathrm{~h}$, portanto, dentro da faixa de temperatura desejada. A temperatura levemente inferior no primeiro horário de avaliação $(8 \mathrm{~h})$ se deve ao fato de que, entre a última avaliação do dia (16 h) e a avaliação às 8 h, não ocorria a abertura da geladeira, assim, explica-se a menor oscilação de temperatura na avaliação às $8 \mathrm{~h}$.

Não houve diferença na motilidade, integridade de membrana e morfologia espermática entre os diluentes e formas de acondicionamento testados até $72 \mathrm{~h}$ (Tabelas 4 e 5) $(\mathrm{p}>0,05)$. Os índices de motilidade e morfologia observados foram superiores a 50 e $80 \%$, respectivamente, e, portanto, dentro das recomendações dos padrões para julgamento de sêmen de doadores da Portaria SDR26 de 05/09/1996 (CBRA, 1998). Ainda que estas recomendações sejam para sêmen refrigerado a 17 ${ }^{\circ} \mathrm{C}$ e os resultados do presente estudo a temperatura de $5{ }^{\circ} \mathrm{C}$. Não há uma recomendação mínima para a integridade de membrana, apesar dos valores encontrados serem compatíveis com outros estudos (VAZQUEZ et al., 1997).

Tabela 4. Percentual de espermatozóides móveis do sêmen suíno congelado por $72 \mathrm{~h}$ a $5{ }^{\circ} \mathrm{C}$, de acordo com o tipo de geladeira utilizada (de sêmen ou convencional) (média \pm desvio padrão).

\begin{tabular}{cccccc}
\hline \multirow{2}{*}{ Diluentes } & \multirow{2}{*}{ Geladeira } & \multicolumn{3}{c}{ Motilidade nos períodos de conservação } \\
\cline { 3 - 5 } & & $\mathbf{0 ~ h}$ & $\mathbf{2 4} \mathbf{~ h}$ & $\mathbf{4 8 ~ h}$ & $\mathbf{7 2 ~ h}^{\text { h }}$ \\
\hline PIGPEL5 & de Sêmen & $76,7 \pm 4,9^{\text {a }}$ & $72,5 \pm 7,5^{\text {a }}$ & $65,8 \pm 9,0^{\text {a }}$ & $60,8 \pm 6,7^{\text {a }}$ \\
PIGPEL5Plus & de Sêmen & $76,7 \pm 4,9^{\text {a }}$ & $70,0 \pm 7,4^{\text {a }}$ & $64,2 \pm 6,7^{\text {a }}$ & $58,3 \pm 7,2^{\text {a }}$ \\
PIGPEL5 & Convencional & $76,7 \pm 4,9^{\text {a }}$ & $70,8 \pm 6,7^{\text {a }}$ & $67,5 \pm 4,5^{\text {a }}$ & $64,2 \pm 6,7^{\text {a }}$ \\
PIGPEL5Plus & Convencional & $76,7 \pm 4,9^{\text {a }}$ & $69,2 \pm 5,2^{\text {a }}$ & $61,7 \pm 5,7^{\text {a }}$ & $58,3 \pm 5,7^{\text {a }}$ \\
\hline
\end{tabular}

Não foram observadas diferenças entre os valores na mesma coluna $(p>0,05)$ 
Tabela 5. Integridade funcional da membrana espermática (CHIPO) do sêmen suíno congelado por $72 \mathrm{~h}$ a $5{ }^{\circ} \mathrm{C}$, de acordo com o tipo de geladeira utilizada (de sêmen ou convencional) (média \pm desvio padrão).

\begin{tabular}{cccccc}
\hline \multirow{2}{*}{ Diluentes } & \multirow{2}{*}{ Geladeira } & \multicolumn{3}{c}{ CHIPO nos períodos de conservação } \\
\cline { 3 - 5 } & & $\mathbf{0 ~ h}$ & $\mathbf{2 4} \mathbf{~ h}$ & $\mathbf{4 8 ~ h}$ & $\mathbf{7 2 ~ \mathbf { ~ }}$ \\
\cline { 3 - 5 } & de Sêmen & $43,4 \pm 5,4^{\mathrm{a}}$ & $40,5 \pm 1,0^{\mathrm{a}}$ & $40,5 \pm 3,3^{\mathrm{a}}$ & $32,6 \pm 3,9^{\mathrm{a}}$ \\
PIGPEL5 & de Sêmen & $43,3 \pm 3,8^{\mathrm{a}}$ & $41,4 \pm 1,9^{\mathrm{a}}$ & $42,8 \pm 2,9^{\mathrm{a}}$ & $31,2 \pm 1,0^{\mathrm{a}}$ \\
PIGPEL5Plus & Convencional & $43,5 \pm 4,7^{\mathrm{a}}$ & $41,8 \pm 1,7^{\mathrm{a}}$ & $41,1 \pm 3,6^{\mathrm{a}}$ & $33,3 \pm 6,6^{\mathrm{a}}$ \\
PIGPEL5 & Convencional & $43,3 \pm 2,9^{\mathrm{a}}$ & $42,2 \pm 3,1^{\mathrm{a}}$ & $42,5 \pm 2,0^{\mathrm{a}}$ & $32,0 \pm 2,1^{\mathrm{a}}$ \\
\hline
\end{tabular}

Não foram observadas diferenças entre os valores na mesma coluna $(p>0,05)$

Com base nos resultados deste experimento observa-se uma vantagem na utilização do diluente PIGPEL5 em geladeira convencional. Isto se deve a maior praticidade na elaboração do diluente com gemadeovo, semnecessidade da adição deprotocolos de purificação de LDL a rotina, diminuindo assim o tempo e custo deste processo. Além do mais, a possibilidade do uso de geladeira convencional, que é de fácil acesso e baixo custo, pode ser vantajosa em pequenas propriedades evitando o investimento em um refrigerador específico para o armazenamento de sêmen.

\section{Conclusões}

O acondicionamento do sêmen no diluente PIGPEL5 em geladeira doméstica $\left(\begin{array}{ll}5 & { }^{\circ} \mathrm{C}\end{array}\right)$ proporcionou padrões de qualidade espermática de acordo com o recomendado para a IA.

\section{Referências}

ALThOUSE, G. C.; LU, K. G. Bacteriospermia in extended porcine semen. Theriogenology, Los Altos, v. 63, n. 2, p. 573-584, 2005.

AMIRAT, L.; TAINTURIER，D.; JEANNEAU，L.; THORIN, C.; GÉRARD, C.; COURTENS, J. L.; ANTON, $\mathrm{M}$. Bull semen in vitro fertility after cryopreservation using egg yolk LDL: a comparison with Optidyl, a commercial egg yolk extender. Theriogenology, Los Altos, v. 61, n. 5, p. 895-907, 2004.

BERGERON, A.; CRÊTE, M. H.; BRINDLE, Y.; MANJUNATH, P. Low-density lipoprotein fraction from hen`s egg yolk decreases the binding of the major protein of bovine seminal plasma to sperm and prevents lipid efflux from the sperm membrane. Biology of Reproduction, Madison, v. 70, n. 3, p. 708-717, 2004.

BOUSSEAU, S.; BRILLARD, J. P.; GUIENNE, B. M.; GUÉRIN, B.; CAMUS, A.; LECHAT, M. Comparison of bacteriological qualities of various egg yolk sources and the in vitro and in vivo fertilizing potential of bovine semen frozen in egg yolk or lecithin based diluents. Theriogenology, Los Altos, v. 50, n. 5, p. 699-706, 1998.

COLÉGIOBRASILEIRODEREPRODUÇÃOANIMAL - CBRA. 2. ed. Manual para exame andrológico e avaliação de sêmen animal. Belo Horizonte: CBRA, 1998.

CORREAA, M. N.; LUCIA JUNIOR, T.; BIANCHI, I.; SCHMITT, E.; BORDIGNON, J.; RECH, D. C.; PERUZZO, I. A.; DESCHAMPS, J. C. Swine semen cooled at $5^{\circ} \mathrm{C}$ with PIGPEL-5 extender: effects on semen quality in vitro and fertility estimators in vivo. Animal Reproduction, Belo Horizonte, v. 3, n. 1, p. 41-48, 2006.

CORRÊA, M. N.; LUCIA JUNIOR, T.; DESCHAMPS, J. C.; SERRET, C. G.; BORDIGNON, J.; RAMBO, G. Taxa de penetração espermática in vitro em ovócitos suínos utilizando espermatozóides acondicionados com o diluente PIGPEL-5 à $5^{\circ} \mathrm{C}$. Revista Brasileira de Reprodução Animal, Belo Horizonte, v. 28, n. 3, p. 161169, 2004.

CORRÊA, M. N.; MEINCKE, W.; LUCIA JUNIOR, T.; DESCHAMPS, J. C. Inseminação Artificial em Suínos. Pelotas: PRINTPAR, 2001.

DEMANIOWICZ, W.; STRZEZEK, J. The effect of lipoprotein fraction from egg yolk on some of the biological properties of boar spermatozoa during storage of the semen in liquid state. Reproduction in Domestics Animals, Berlin, v. 31, n. 3, p. 279-280, 1996.

GRAHAM, J. K.; FOOTE, R. H. Effect of several lipids fatty acil chain length and degree of unsaturation on the 
motility of bull spermatozoa after cold shock and freezing. Criobiology, Hamburg, v. 24, n. 1, p. 42-52, 1987.

HU, J. H.; LI, Q. W.; LI, G.; CHEN, X. Y.; YANG, H.; ZHANG, S. S.; WANG, L. Q. The cryoprotective effect on frozen-thawed boar semen of egg yolk low density lipoproteins. Asian-Australian Journal of Animal Science, Seul, v. 19, n. 4, p. 486-490, 2006.

JOHNSON, L.A.; WEITZE, K. F.; FISER, P.; MAXWELL W. M. C. Storage of boar semen. Animal Reproduction Science, Amsterdan, v. 62, n. 1/3, p. 143-172. 2000.

LEVIS, D. G. Liquid boar semen production: current extender technology and where we go from here. In: INTERNATIONALCONFERENCE ON BOAR SEMEN PRESERVATION, 4., 2000, Beltsville. Proceedings... Beltsville, 2000. p. 121-128.

MOUSSA, M.; MARTINET, V.; TRIMECHE, A.; TAINTURIER, D.; ANTON, M. Low density lipoproteins extracted from hen egg yolk by an easy method: cryoprotective effect on frozen-thawed bull semen. Theriogenology, Los Altos, v. 57, n. 6, p. 16951706, 2002.

PURSEL, V. G.; JOHNSON, L. A. Freezing of boar spermatozoa: Fertilizing capacity with concentrated semen and a new thawing procedure. Journal of Animal Science, Champaign, v. 40, n. 1, p. 99-102, 1975.

PURSEL, V. G.; JOHNSON, L. A.; RAMPACEK, G. B. Acrosome morphology of boar spermatozoa incubated before cold shock. Journal of Animal Science, Champaign, v. 34, n. 2, p. 278-283, 1972.

PURSEL, V. G.; JOHNSON, L. A.; SCHULMAN, L. L. Interaction of extender composition and incubation period on cold shock susceptibility of boar spermatozoa. Journal of Animal Science, Champaign, v. 35, n. 3, p. 580-584, 1972.

PURSEL, V. G.; SCHULMAN, L. L.; JOHNSON, L. A. Effect of holding time on storage of boar spermatozoa at $5^{\circ}$ C. Journal of Animal Science, Champaign, v. 37, n. 3, p. 785-789, 1973.
STATISTIX $^{\circledR}$ 8. Analytical Software. Tallahassee: FL, 2003.

TARDIF, S.; LAFOREST, J. P.; CORMIER, N.; BAILEY, J. L. The importance of porcine sperm parameters on fertility in vivo. Theriogenology, Los Altos, v. 52, n. 3, p. 447-459, 1999.

TRIMECHE, A.; ANTON, M.; RENARD, P.; GANDEMER, G.; TAINTURIER, D. Quail egg yolk: A novel crioprotectant fot the freeze preservation of Poitou jackass sperm. Criobiology, Hamburg, v. 34, n. 4, p. 385393, 1996.

VARELA JUNIOR, A. S.; CORCINI, C. D.; ULGUIM, R. R.; ALVARENGA, M. V. F.; BIANCHI, I.; CORRÊA, M. N.; LUCIA JUNIOR, T.; DESCHAMPS, J. C. Effect of low density lipoprotein on the quality of cryopreserved dog semen. Animal Reproduction Science, Amsterdan, v. 115, n. 1/4, p. 323-327, 2008.

VAZQUEZ, J. M.; MARTINEZ, E. A.; MARTINEZ, P.; GARCIA-ARTIGA, C.; ROCA, J. Hypoosmotic swelling of boar spermatozoa compared to other methods for analysing the sperm membrane. Theriogenology, Los Altos, v. 47, n. 3, p. 913-922, 1997.

WATSON, P. F. The roles of lipid and protein in the protection of ram spermatozoa at 5 degrees $\mathrm{C}$ by eggyolk lipoprotein. Journal of Reproduction and Fertility, Cambridge, v. 62, n. 2, p. 483-492, 1981.

WATSON, P. F. The causes of reduced fertility with cryopreserved semen. Animal Reproduction Science, Amsterdan, v. 60/61, n. 1, p. 481-492, 2000.

WOELDERS, H. Overview of in vitro methods for evaluation of semen quality. In: INTERNATIONAL CONFERENCE ON BOAR SEMEN PRESERVATION, 2., 1991, Berlin. Proceedings... Berlin, 1991. p. 145164. 